\title{
Impacting cancer survivorship
}

\author{
Michael Feuerstein
}

Received: 16 July 2012 / Accepted: 24 July 2012 / Published online: 10 August 2012

(C) Springer Science+Business Media, LLC (outside the USA) 2012

Regardless of the controversy over just what is a cancer survivor, improving the health and daily lives of cancer survivors is of paramount importance. The Journal of Cancer Survivorship: Research and Practice (JCS) was created to help achieve these goals. I have a few announcements that highlight the progress we have made thus far.

The journal has reached an important milestone. JCS has received its initial impact factor. It is with great excitement that we can say evidence exists that JCS, the first journal devoted exclusively to the dissemination of knowledge related to improving the health, health care, function, and well-being of cancer survivors, has been accepted by the scientific community (Science Citation Index Expanded, Journal Citation Reports/Science Edition, Current Contents/Clinical Medicine, 2011). The impact factor for JCS, which represents citations of papers from the journal by other scientists, over a 2-year period (2009-2010) was a respectable 2.629 (Thomson Reuters Journal Citation Reports $\left.{ }^{\circledR}, 2011\right)$.

We all should be very pleased with these results. I want to take this opportunity to thank all of you who contributed papers, reviewed submissions, and helped process manuscripts to make this information available to researchers, practitioners, cancer survivors, and students pursuing a range of careers. Also thanks to all the editorial board members who agreed to participate in this nascent adventure 6 years ago and to the editors at Springer who believed me when I told them of the need for such a journal.

\section{Development}

The journal is now 5 years old. Your trust in the mission of $J C S$ to improve what we know about long-term and late

M. Feuerstein $(\bowtie)$

Boston, MA, USA

e-mail: jcancersurvivorship@gmail.com outcomes and to improve the health and lives of those surviving a cancer diagnosis and treatment has powered this effort. JCS has a strong foundation. In its first year, the journal was indexed in major search engines within science and health including the search systems of the U.S. Library of Medicine. Subsequent years saw a steady increase in the quality and breath of submissions. As more and more papers were received, in order to vary the topics, methods, and professions actively involved in cancer survivorship, the number of papers that could be reasonably accepted continued to decrease to a level where we are currently accepting three papers for every ten that are submitted. There is no plan to make JCS a monthly periodical. Papers are published and indexed online in a very timely manner. This process provides rapid access to many and immediate citation. Therefore, there is very little justification to increase the number of print issues. However, if someone or some group is considering publication of a special issue of the journal, we are always interested in discussing this with you.

\section{Impact for the long term}

We seek submissions from all researchers or research clinicians involved in any aspect of cancer survivorship that fits with the mission. However, for those of you in the early stages of your careers, especially consider JCS for publication of your work. If your submission is consistent with the mission of the journal, is of high quality, and presents new knowledge of interest to researchers, clinicians, or survivors, if necessary, we will provide you with feedback that should help you make the information even more accessible rather than rejecting the paper. We want you to be successful. You are the future of cancer survivorship research and practice. Also, Springer will facilitate wider dissemination of select papers. This no cost public relations service helps get the word out to many stakeholders who 
may not typically be aware of your work. In the age of Internet communication, this is even more critical than ever before. While these public media releases will not earn you any academic "points," they do increase the dissemination of your work and can influence the uptake of this new knowledge.

\section{Preventing bottlenecks}

Fortunately JCS is receiving ever-increasing numbers of manuscripts. It is now time to ask for help triaging manuscripts through review, revision, and production. These "Associate Editors" will be responsible for specific content areas including health care (oncology, primary care medicine, nursing, and psychiatry/psychology/social work), lifestyle (health behavior change), epidemiology (descriptive epidemiology, complex quantitative analyses/biostatistics, and methodology), health services (access, cost, and quality), economics, function (rehabilitation), functional restoration (physical medicine and physical therapy), work, qualitative methods, symptom burden (fatigue, pain, cognitive limitations, and depression), communication, decision making, integrative health care, self-management, young adult cancer survivors, and well-being. This list is not all inclusive. Recommendations of topic areas not covered above are welcome. There will be a group of Associate Editors who will work closely with the editor and editorial board to process submissions in a professional, supportive, and efficient manner. Please consider colleagues or yourselves for these volunteer positions. This activity should not require more than $5 \mathrm{~h} /$ week for any one person.

\section{The future}

It is expected that there will be a shortage of physicians, nurses, physician extenders, and other health care providers over the next few decades to meet the demands of cancer patients and cancer survivors. These health professionals are needed to address the many challenges of cancer survivors, and their shortage is expected to create a "perfect storm" (i.e., increase in the number of cancer survivors/shortage of providers) with major public health implications. Publishing knowledge that can be used to generate solutions to this anticipated crisis represents a top priority of this journal. If we can prevent this crisis or more likely reduce its severity, we will be much better off than playing catch up. While prevention is often an elusive goal, the knowledge disseminated in JCS over the next decade or two should reduce the severity of this storm. If this happens, then we can really say $J C S$ has made an impact. 